\title{
Antenna Interconnection Strategies for Personal Communication Systems
}

\author{
Halim Yanikomeroglu and Elvino S. Sousa, Senior Member, IEEE
}

\begin{abstract}
Microcellular and distributed antenna systems are two promising candidates for implementing personal communication systems. In this paper, antenna interconnection strategies for these systems are studied in order to determine cost-efficient as well as robust and flexible architectures in hexagonal layouts. To this end, some results from minimal networks theory are used, in particular, those dealing with the problem of Steiner trees. The significant reduction in conduit and cable lengths that the Steiner minimal tree (SMT) architecture provides over the star type, especially in large networks, is demonstrated. It is further shown that the SMT architecture also provides more flexibility and robustness compared to the star type. The suboptimal, but easy-to-construct, minimal spanning tree (MST) architecture is given as well, and it is compared to the SMT and star types.
\end{abstract}

Index Terms - CDMA, cellular systems, distributed antennas, leaky feeder antennas, optical fiber systems, personal communication networks, Steiner trees.

\section{INTRODUCTION}

A CCESS networks for personal communication systems (PCS's) interact with existing fixed networks (public switched telephone network (PSTN) or other broad-band networks) to extend information services to wireless terminals. In order to provide high-capacity, ubiquitous coverage, and to be able to support compact and lightweight wireless terminals, a very large number of cells should be deployed in urban areas; according to one estimate, more than 5000 cells would be required in the Tokyo metropolitan area [1]. Therefore, building an affordable infrastructure for the access network of PCS is a major challenge.

In the current cellular mobile communication systems (such as AMPS or GSM), T1 lines or microwave radio links are most commonly used for connecting the base stations (BS's) to the mobile telephone switching office (MTSO) [2]. In the case of the $\mathrm{T} 1$ cables, generally, the star architecture is employed, mainly due to its topological simplicity. In a star architecture, if a new BS is to be added to the network, a cable is laid down between the new BS and MTSO. Hence, there is no need for coordination with the other BS's. Also, a fault in any BS, or in the cable, would only affect that particular BS, and moreover, the distance between any BS and the MTSO is minimal, which may eliminate the need for amplifiers in the feeder. However, the required cabling for the star architecture is excessive.

Manuscript received May 29, 1996; revised April 15, 1997. This work was supported by a grant from the Canadian Institute for Telecommunications Research under the NCE Program of the Government of Canada.

The authors are with the Department of Electrical and Computer Engineering, University of Toronto, Toronto, Ont. M5S 3G4, Canada.

Publisher Item Identifier S 0733-8716(97)05848-4.
In a system which consists of a few base stations and a switching office, it may not matter how the interconnection of these network elements is made. But, if there is an extensive network with thousands of cells and numerous processors/switches, then it may be crucial to have a strategy or algorithm to achieve the interconnection in an efficient manner because even modest improvements in the design of the PCS access network would result in significant savings. In this paper, antenna interconnection strategies for wireless access networks are studied in order to determine cost-efficient as well as robust and flexible interconnection architectures. To this end, some results from minimal networks theory are used, in particular, those dealing with the well-known problem of Steiner trees.

In Section II, an overview of PCS access networks is presented. In Section III, the Steiner minimal tree (SMT) architecture is described, and SMT construction in hexagonal layouts is discussed. The conduit length comparisons between the star and SMT architectures are presented in Section IV. In Section V, interconnection strategies for linking various central stations are examined. The realization of logical network topologies within the optimal SMT conduit infrastructure is addressed in Section VI; and finally, concluding remarks are made in Section VII.

\section{PCS ACCESS NETWORK}

In this section, an overview of the two promising candidates for the PCS access network, namely, microcellular and distributed antenna systems, is given, and the logical topologies corresponding to these systems are discussed.

\section{A. Antenna Interconnection in Microcellular Systems}

The natural extension of the cellular concept has led to the proposal of microcellular systems for the PCS access network [3], [4]. Since the radius of a microcell is on the order of a few hundred meters or less, a very large number of microcells will be required to cover metropolitan areas. If a conventional base station is needed for each microcell, then the deployment cost would become extremely high. Furthermore, it is very difficult to find convenient locations for the bulky base station equipment every few hundred meters in urban areas. Therefore, it would be very desirable if the microcell base station (which will be referred to as Micro-BS from now on) could contain a minimal amount of hardware, and if most of the processing could be performed at a central station (CS) located at a convenient economical site, serving many of these microcells. 
The different possible methods of distributing the processing among a Micro-BS and the CS yield a variety of systems. Ultimately, all of the signal-specific processing can be moved to the CS, from which the radio signals are delivered to MicroBS's via fiber optic, coaxial, or microwave links [4], [5].

In a metropolitan area where the Micro-BS's are many kilometers away from the CS, it is almost impossible to have line-of-sight links between the utility-post-mounted Micro-BS antennas and the CS. This would yield severe multipath and shadowing phenomena in addition to the path loss. Therefore, despite their easy installation, microwave links are not suitable in many environments (although the networking of CS's may be realized via microwave links, as we will address in Section V). The main problems associated with coaxial links are the significant losses in the cable and the poor frequency response [5]. Even if high-gain RF amplifiers are inserted in the feeder, the resulting SNR is not sufficient, unless the distances between the CS and Micro-BS's are short enough to utilize repeaterless coaxial cables [6].

On the other hand, advances in lightwave technologies, along with the availability of wide-bandwidth, very lowloss (around to $0.2 \mathrm{~dB} / \mathrm{km}$ ) fiber optic cables, enable the tranmission of radio signals between the CS and Micro-BS's without amplification for more than $20 \mathrm{~km}$, using subcarrier multiplexing (SCM) [1], [7]. In this case, the Micro-BS would contain only an optoelectric $(\mathrm{O} / \mathrm{E})$ converter and power amplifier (PA) in the forward link, and a low-noise amplifier (LNA) and an electrooptic (E/O) converter in the reverse link, as shown in Fig. 1(a). ${ }^{1}$ The fiber optic antenna remoting offers other advantages along with simple and economical Micro-BS's, for instance, centralized control (which is suitable for macro diversity and dynamic channel allocation), and transparency to the modulation scheme (FDMA, TDMA, or CDMA) employed [2].

The above described system has a logical star topology because of the point-to-point nature of SCM fiber optic links [Fig. 1(a)] [5], [8]-[10]. The star architecture provides high reliability and easy maintenance due to its topology; however, it requires an enormous investment for the wired network infrastucture. In order to decrease the infrastucture cost, an alternative architecture, the passive double star (PDS), has been proposed which employs optical passive splitters, as illustrated in Fig. 1(b) [10]. Although the PDS architecture yields great savings in wired network infrastucture, the simplicity of Micro-BS, which was originally one of the major motivations for using fiber optic links, is lost. This is due to the fact that, since the PDS architecture has a logical bus topology, the CS cannot distinguish between the signals coming from different Micro-BS's unless Micro-BS's are equipped with transmitter/receivers (TX/RX) and modems.

One other possibility is to implement a bus architecture, in which case many Micro-BS's are coupled to a fiber optic bus via passive optical couplers as shown in Fig. 1(c), and timedivision multiplexing (TDM) is employed to distinguish the

\footnotetext{
${ }^{1}$ This concept has been addressed in the literature under different names, such as "antenna remoting" [2], "extension," or "separation" [5], and the Micro-BS has been referred to as "remote cell site" [2], "microcell base unit" [3], "antenna remote unit" (ARU) [7], and "radio base station" (RBS) [8].
}

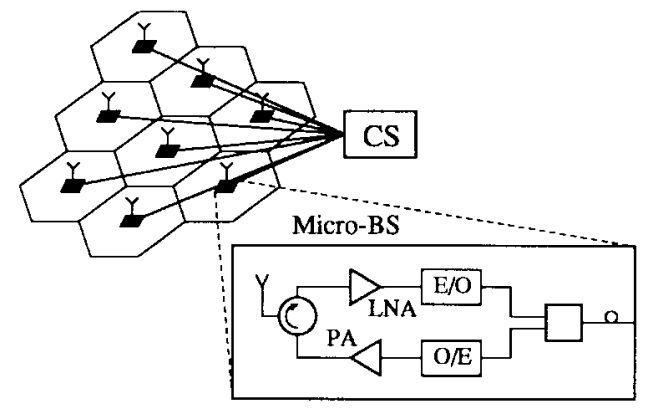

(a)



(b)

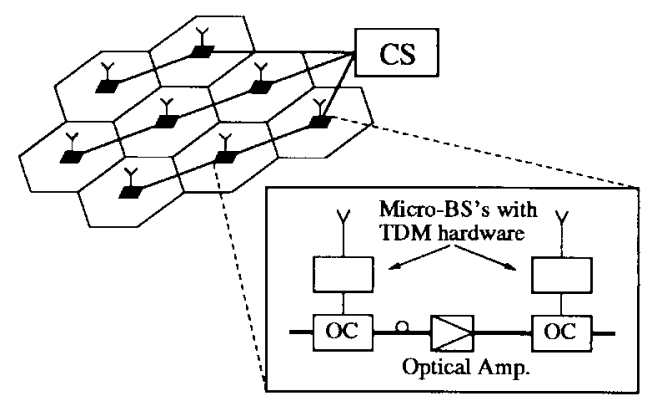

(c)

Fig. 1. Microcellular access networks for PCS using optical fiber. (a) SCM links (logical star topology). (b) Double-star links (logical bus topology). (c) TDM links (logical bus topology).

signals of different Micro-BS's [11]. In this scheme, however, to compensate for the coupling losses, optical amplifiers should be inserted in the cable, and furthermore, hardware should be included at Micro-BS's for TDM. An amplifier chain in the feeder is not desirable since, in such a case, the Micro-BS's that are further away from the CS have much poorer reliability compared to those which are closer.

Hybrid coaxial/SCM-fiber links can also be utilized for the PCS access network [6]. In this system, the Micro-BS's which belong to the same cluster of microcells can be connected to a signal combiner via coaxial cables (as long as the coaxial cable length is less than a few hundred meters so that there is no need for amplification in the feeder), and the combiner is connected to the CS via a fiber optic link. Since the signals from all of the microcells in a cluster are summed at the combiner, this architecture, which is suitable for FDMA and TDMA schemes, cannot be used with the CDMA scheme. We note that the 
hybrid coaxial/fiber network is also a double-star architecture (with a small splitting ratio), and therefore it has a logical bus topology.

\section{B. Distributed Antenna and Sectorized Distributed Antenna Systems}

One problem associated with the small cell size is maintaining the link control. Ideally, handoffs should not occur frequently; yet, if they do, the system should be able to readily cope with them. Furthermore, there are environments where user density is relatively low, yet extensive coverage is still required. For such environments, distributed antenna (DA) systems have been proposed as the access network for PCS's [12]-[15].

In a DA cell, many simple antenna elements are coupled to a common feeder. The same signal is transmitted from (and received by) all of the antenna elements which are distributed throughout the cell; therefore, as long as the wireless user is in the same cell, there is no need for handoff. There is no processing at the antenna elements except for amplification; all of the signal-specific processing is done at the CS. In order to exploit self-interference in the overlapping regions of a DA cell, CDMA modulation is employed and delay elements are inserted in the feeder to guarantee that the received signals from different antenna elements are at least one PN (pseudonoise) code chip period apart; this enables the Rake receiver to distinguish between the signals picked up by different antenna elements. The DA system has many other appealing features: the macro diversity effect against shadow fading, transmission with relatively low transmit power levels, less interference to other systems, uniform coverage of a service area, and formation of cells with desirable shapes, even noncontiguous ones [16].

The drawback of the DA system is that the capacity per antenna element is low as a result of the multiple-access interference accumulated in the feeder. To overcome the capacity limitation, a scheme called the sectorized distributed antenna (SDA) is proposed [16]. In the SDA scheme, a cell has many sectors in which separate feeders run, so multiple-access interference is reduced, and thus the capacity is increased. In a sector, there might be more than one antenna element, and between sectors, mobile transparent handoff is performed. In the limiting case of one antenna element per sector, a wireless user's signal can be picked up by all of the antenna elements in an SDA cell, and then can be optimally combined at the CS. This scheme, which can be used both indoors and outdoors, overcomes the capacity limitations and further increases the SNR (signal-to-noise ratio). Research analyzing the performance of this novel system is in progress.

Both DA and SDA systems have logical bus topologies. In the case of a DA scheme, a coaxial bus link can be used to connect the antenna elements and the CS, as long as the cell is not very large; obviously, a fiber optic bus employing SCM is another possibility. In either case, amplifiers may need to be inserted in the feeder to compensate for the coupling losses. It is worth noting that, unlike the microcellular bus link discussed in the previous section, the DA bus link does not require the

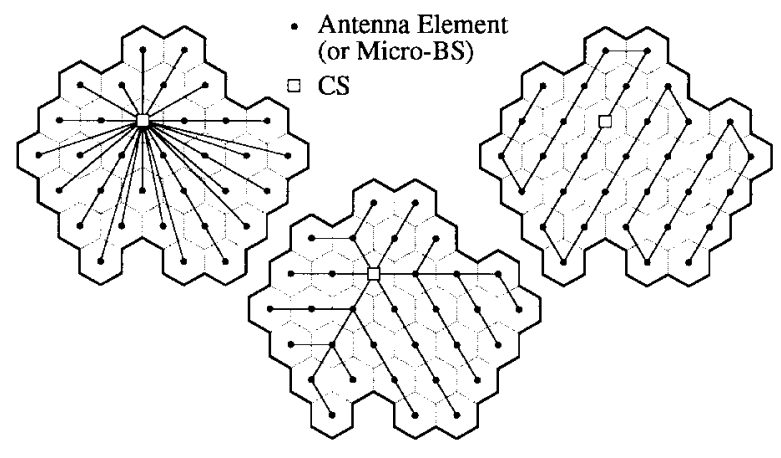

(a)

(b)

(c)

Fig. 2. (a) Star and (b)-(c) MST architectures in hexagonal layout.

use of TDM or frequency conversions at the antenna elements since, in a DA cell, the same signal is fed to all of the antenna elements.

In the case of an SDA, a separate bus link runs in each sector, which may be either a coaxial or a fiber optic cable, although the fiber may be more appropriate for the farther sectors. A hybrid coaxial/fiber bus link may be another possibility; the radio signal may be delivered to a distant sector via a fiber cable, and in the sector, a coaxial bus may be used. For the limiting case of one antenna element per sector in an SDA, the architecture is the star type, and transmission without repeaters is possible since there are no coupling losses.

\section{Logical Topology Versus Conduit Structure}

A cable can be installed only on those links of a wired network where a conduit has been placed. Since the conduit placement is far more expensive than the material cost of the cable, the cost-saving cable deployment strategy is to let a conduit path be shared by many different cable routes wherever possible [17]. For instance, in a microcellular network which has logical star topology, there is only one cable route between the CS and a Micro-BS; however, the connections are not necessarily restricted to the "point-to-point" type. In other words, the logical topology and the actual conduit layout do not need to have the same architecture. In the following few sections, the focus will be on constructing efficient conduit architectures, and in Section VI, a discussion will be presented on how to map a logical cable topology into the optimal conduit infrastructure.

\section{The Steiner Minimal Tree Architecture}

Let us consider a typical access network where many antenna elements (or Micro-BS's) are to be connected to a CS. The straightforward and conventional interconnection structure is the star architecture as shown in Fig. 2(a) for the hexagonal layout. However, as mentioned earlier, the main problem in implementing this architecture is the enormous financial investment required for the construction of the conduit infrastructure.

The architectures illustrated in Fig. 2 may correspond to DA (or SDA) systems with antenna elements and CS's, or to microcellular systems with Micro-BS's and CS's. Without loss of generality, however, we will assume that the architectures 
studied in this paper correspond to DA systems. Thus, a hexagonal region shown by the dotted lines in Fig. 2 (and also in other figures) does not denote an individual cell; rather, it is the area wherein a wireless user is most likely to receive the strongest signal from the corresponding antenna element in the center of the region. A cell, then, refers to the area bordered by thick lines in the figures that encloses many antenna elements and a CS. On the other hand, for the case of a microcellular system, this bordered area would correspond to a service area covered by many Micro-BS's connected to a CS.

\section{A. Shortest Interconnection Network Problem}

A network architecture which consists of antenna elements and transmission links can be represented by a connected graph where the antenna elements are shown by vertices (points) and the links by edges. Let $P=\left\{P_{1}, P_{2}, \cdots, P_{N}\right\}$ be a set of $N$ points in the plane, denoting $N$ antenna elements to be interconnected. Since the objective is to minimize the total conduit length, the corresponding graph that interconnects these $N$ points should not contain any cycles because removing an edge from the cycle will readily reduce the total length (or weight), but this will not disconnect the graph. Therefore, a tree structure that spans all of the vertices and also yields the minimum total length is required.

If no new points are allowed to be added to the original set $P$ (i.e., if the vertices of the required tree are exactly the points in $P)$, then the shortest network connecting these points is called a minimal spanning tree (MST). Two classical algorithms for finding such a tree are Prim's algorithm (1957), which adds edges which extend the existing tree and do not create cycles, and Kruskal's algorithm (1956), which adds edges which connect components [18, pp. 593-598]. In Fig. 2(b) and (c), two different MST's in a hexagonal layout are illustrated. We note that since the lengths (weights) of the edges in the hexagonal layout are not all different, MST is not unique.

However, it is possible to construct still shorter trees connecting $P_{1}, \cdots, P_{N}$ by adding extra vertices beside the $P_{i}$. The shortest possible tree constructed in this way is called the Steiner minimal tree (SMT), and the additional vertices are called Steiner points [19], [20].

A tree interconnecting a set of $N$ points in the plane, by adding extra vertices (Steiner points) if necessary, is called a Steiner tree (ST) if it satisfies the following conditions [21].

1) No two edges meet at less than $120^{\circ}$.

2) There are at most $N-2$ Steiner points.

3) Each Steiner point has exactly three incident edges.

Conditions 1) and 3) together imply that every Steiner point has exactly three edges meeting at $120^{\circ}$. An SMT is simply the shortest ST; thus, it should be emphasized that not every ST is an SMT. Finally, an ST is called full (FST) if it has exactly $N-2$ Steiner points.

\section{B. SMT Construction Principles}

The SMT constructions for $N=3$ and $N=4$ are well known. A summary is presented below for these cases along with the general case of $N$ points.

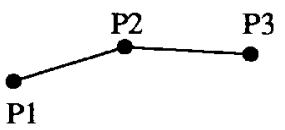

(a)

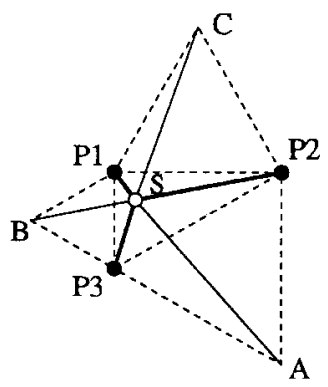

(b)



(d)

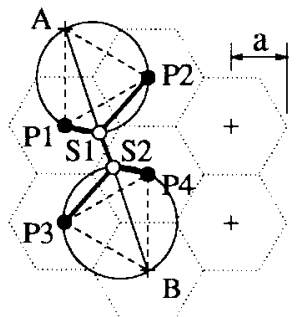

(f)

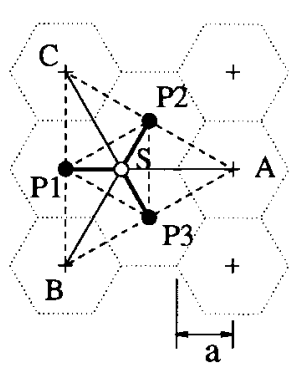

(c)

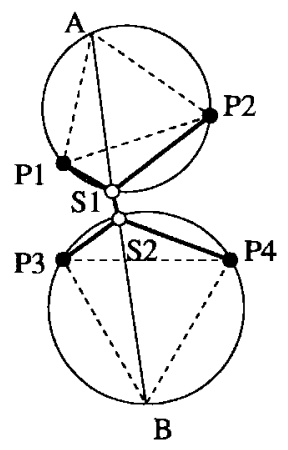

(e)

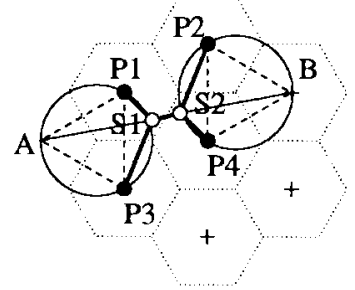

(g)
Fig. 3. (a) $N=3$, general case, $\triangle P_{1} P_{2} P_{3}$ has an angle $\geq 120^{\circ}$. (b) $N=3$, general case, $\triangle P_{1} P_{2} P_{3}$ has no angles $\geq 120^{\circ}$. (c) $N=3$, the special case of hexagonal layout. (d) $N=4$, general case. (e) $N=4$, general case, an ST (not an SMT). (f) and (g) $N=4$, the special case of hexagonal layout.

$N=3:$ If $\triangle P_{1} P_{2} P_{3}$ has an angle $\geq 120^{\circ}$, then adding Steiner points does not help in reducing the total length of the network [Fig. 3(a)]. Otherwise, three equilateral triangles are drawn on the sides of $\triangle P_{1} P_{2} P_{3}$. If the far vertex of each equilateral triangle is joined to the opposite $P_{i}$, then these lines meet at a single Steiner point ${ }^{2}$ [Fig. 3(b)]. The length of the SMT constructed in this way, $L_{\mathrm{SMT}}=P_{1} S+P_{2} S+P_{3} S$, satisfies [22, p. 140]

$$
L_{\mathrm{SMT}}=P_{1} A=P_{2} B=P_{3} C .
$$

For the special case of the hexagonal layout, with hexagon radius $a$, it is obvious from Fig. 3(c) that

$$
L_{\mathrm{SMT}}=3 a \text {. }
$$

\footnotetext{
${ }^{2}$ In Figs. 3 and 4, the Steiner points are shown by white dots.
} 
$N=4$ : Similar to the case for $N=3$, equilateral triangles are drawn on the sides $P_{1} P_{3}$ and $P_{2} P_{4}$ [Fig. 3(d)], and then their far vertices ( $A$ and $B$, respectively) are joined. The intersection of this line and the circles shown locates Steiner points $S_{1}$ and $S_{2}$. It can be shown [24] that, in Fig. 3(d)

$$
P_{1} S_{1}+P_{3} S_{1}=A S_{1} \quad \text { and } \quad P_{2} S_{2}+P_{4} S_{2}=B S_{2} .
$$

Therefore, the length of the SMT with vertices $P_{1}, P_{2}, P_{3}, P_{4}$, $S_{1}$, and $S_{2}$ is equal to $A B$. We note that another ST can be formed [Fig. 3(e)] in general; however, the ST with the longer central edge is the SMT. For the special case of hexagonal layout [Fig. 3(f) and (g)], $L_{\mathrm{SMT}}(=A B)$ is the hypotenuse of a right triangle whose other two sides have lengths $4.5 \mathrm{a}$ and $\sqrt{3} a / 2$; so using the Pythagorean theorem, $L_{\mathrm{SMT}}$ can be shown as

$$
L_{\mathrm{SMT}}=\sqrt{(4.5 a)^{2}+(\sqrt{3} a / 2)^{2}}=\sqrt{21} a .
$$

It is worth noting that for the hexagonal layout, both of the possible ST's [Fig. 3(f) and (g)] are SMT's.

$N$-Point Case: If $N \geq 5$, then SMT construction becomes very difficult. The existence of a finite algorithm for SMT construction was proven by Melzak in 1961 [23]; but this problem in the general case is extremely complicated. Melzak's algorithm makes use of the following observation: if the SMT does not have as many as $N-2$ Steiner points, then the SMT will decompose into a number of smaller FST's which meet each other at some subset of the given vertices [24]. Melzak's algorithm makes use of the following facts [25]: SMT $(P)$ (which denotes the SMT corresponding to set $P$ ) may always be decomposed into sets $\operatorname{SMT}\left(P_{1}\right), \operatorname{SMT}\left(P_{2}\right), \cdots, \operatorname{SMT}\left(P_{l}\right)$, where $P_{1}, P_{2}, \cdots, P_{l}$ are subsets of $P$ with $\left|P_{i} \cap P_{j}\right| \leq 1 \forall i \neq$ $j$, where $\operatorname{SMT}\left(P_{k}\right)$ is an FST for $P_{k}$, and the edges of the $\operatorname{SMT}\left(P_{k}\right)$ form a partition of the edges of $\operatorname{SMT}(P)$. Fig. 4(a) and $(b)^{3}$ shows such a decomposition and the yielding SMT, respectively, for a case of $N=10$. Although we do not know a priori how to decompose the given $N$ points into subsets, the total number of possible decompositions is finite [22]. However, if we try all possibilities, their number will grow exponentially. The exponentiality is more of a problem inherent to the SMT rather than just to Melzak's algorithm [20]; indeed, it is shown in [26] that the SMT construction for a general set $P$ is NP-complete.

\section{SMT Construction for Hexagonal Layout}

An important consideration for NP-complete problems is the study of special cases. To this end, in this section, we discuss the SMT construction for hexagonal layout. We note the practical importance of this layout since the hexagon is the most commonly used geometrical shape in modeling the service area of an antenna element in wireless communications.

Let us first introduce the concept of the Steiner ratio. For a given arbitrary set of points $P$, if $L_{\mathrm{MST}}$ and $L_{\mathrm{SMT}}$ denote the lengths of the MST and SMT, respectively, then the following theorem holds (for the proof, see [19, pp. 166-177]).

\footnotetext{
${ }^{3}$ Fig. 4(b) is taken from [22, p. 142].
}

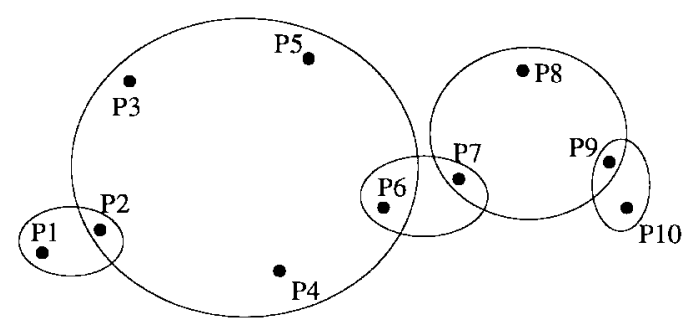

(a)

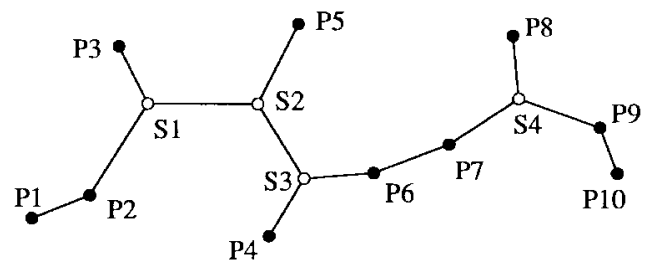

(b)

Fig. 4. SMT construction for a case of $N=10$. (a) Decomposition. (b) Yielding SMT.

Theorem 1: $\rho=\inf _{P}\left(L_{\mathrm{SMT}}(P) / L_{\mathrm{MST}}(P)\right)=\sqrt{3} / 2=$ $0.8660 \cdots$, where "inf" is taken over all possible sets of $P$. In the above, $\rho$ is called the Steiner ratio.

We note that it is quite important to know to what extent a heuristic (like MST) approximates the optimum structure (SMT) since the problem of SMT construction is NP-complete. Since, for any layout

$$
\rho=\frac{\sqrt{3}}{2}=0.8660 \leq \frac{L_{\mathrm{SMT}}}{L_{\mathrm{MST}}} \leq 1
$$

the maximum increase in conduit length for constructing an MST instead of the SMT can be calculated as

$$
\frac{1-\sqrt{3} / 2}{\sqrt{3} / 2} \times 100 \% \simeq 15 \% \text {. }
$$

1) Hexagonal Cells: It is shown in [27] by induction that for the special case of a hexagonal cell in the hexagonal layout, the SMT construction is achieved by decomposing the vertices into subsets of three points which form equilateral triangles; here, we will give an alternate proof based on the concept of the Steiner ratio. Since the vertices in the hexagonal layout are located at the intersection points of the triangular grid, this decomposing strategy is intuitively satisfying. There are many possible ways of forming these three-point subsets; one of them, along with the corresponding SMT, is illustrated in Fig. 5.

It can be shown that in a hexagonal cell constructed in the hexagonal layout, for a given $n$ (which is the number of antennas at one side of the hexagonal cell), there are

$$
N=3 n(n-1)+1
$$

antennas (points), for instance, in Fig. 5, $n=4$ and $N=37$.

Let $L_{0}$ be the length of the tree structure illustrated in Fig. 5 . It is not difficult to show that there are a total of $(N-1) / 2$ three-point subsets in such a tree with $N$ points. Since the three-point FST's formed for each subset have lengths of $3 a$

$$
L_{0}=\frac{N-1}{2} 3 a \text {. }
$$




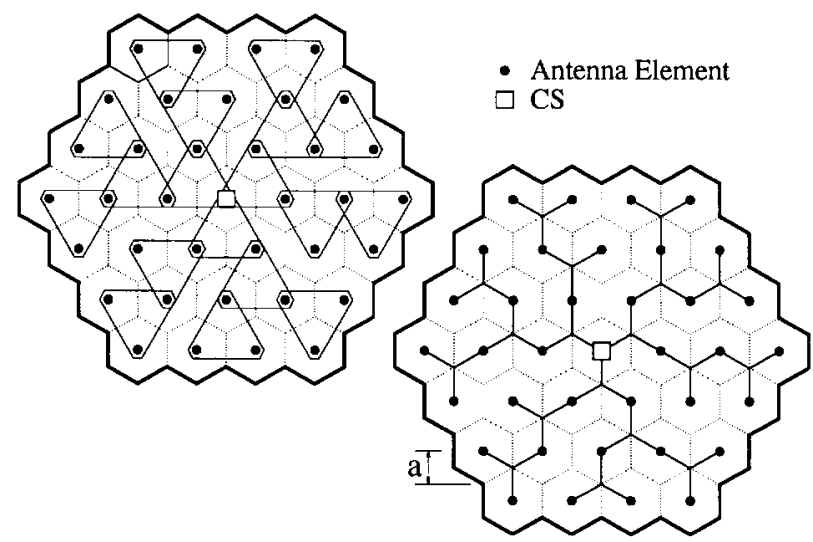

Fig. 5. SMT construction in hexagonal layout: decomposition and the resultant SMT.

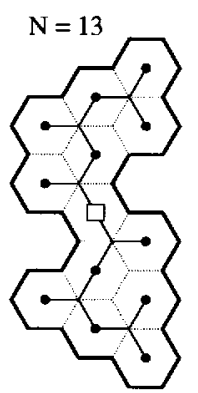

(a)

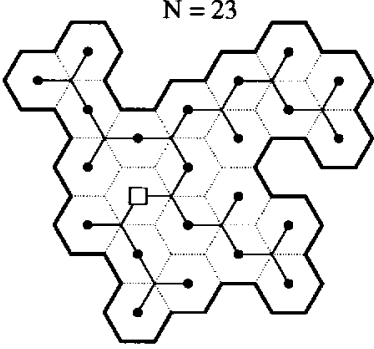

(b)
- Antenna Element $\square \mathrm{CS}$



(c)
Fig. 6. SMT's for general cell shapes in a hexagonal layout. (a) $N=13$. (b) $N=23$. (c) $N=5$.

It is well known that if a graph is a tree with $N$ vertices, then it has precisely $N-1$ edges. In the hexagonal layout with hexagon radius $a$, the distance between any two neighboring vertices, which is the length of an edge of the MST, is found to be $\sqrt{3} a$. Therefore, $L_{\mathrm{MST}}$ for an $N$-point hexagonal cell [see Fig. 2(b) and (c)] is

$$
L_{\mathrm{MST}}=(N-1) \sqrt{3} a .
$$

If the ratio of the above two equations is taken, we obtain

$$
L_{0} / L_{\mathrm{MST}}=\frac{\sqrt{3}}{2} \text {. }
$$

But this is equal to $\rho$, the Steiner ratio; therefore, $L_{\mathrm{SMT}}=L_{0}$. This result shows that the decomposition strategy in the form of three-point subsets depicted in Fig. 5 yields SMT's.

2) General Cell Shape: Based on the proof given in the previous section, many other interconnecting structures for arbitrary cell shapes can also be shown to be SMT's as long as $N$ is an odd number and the exact decomposition of set $P$ into three-point subsets is possible, as shown in Fig. 6(a) and (b). If $N$ is an even number, however, this exact decomposition into subsets of three-points cannot be realized. Even for an odd $N$, there may be cell shapes for which subsets with a number of points other than three may need to be formed; an example is shown in Fig. 6(c) where two-point subsets along with a three-point one are formed to construct the SMT. For such cases, where the exact decomposition into subsets of threepoints is not possible, however, we can not use the Steiner

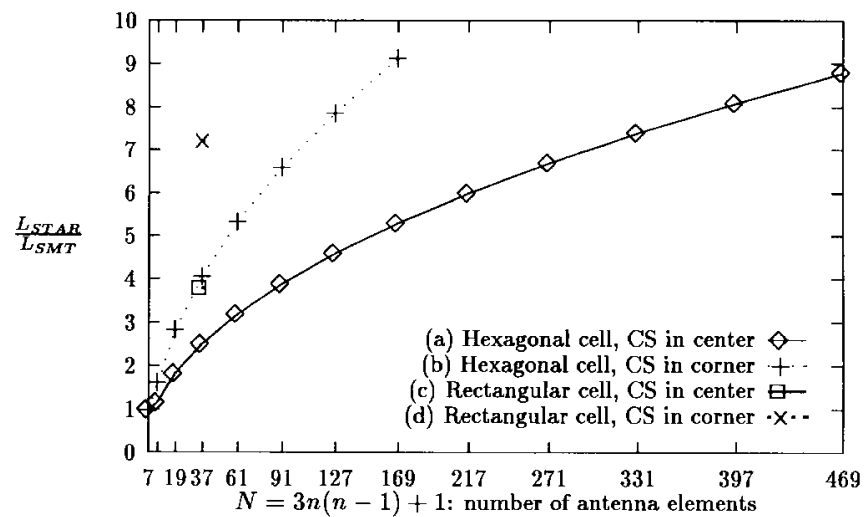

Fig. 7. Conduit length comparisons between star and SMT architectures.

ratio theorem to prove that these interconnecting structures are indeed SMT's.

Because of the fact stated in (5), we can be certain that whatever the cell shape is

$$
\rho L_{\mathrm{MST}}=\frac{3}{2}(N-1) a \leq L_{\mathrm{SMT}} \leq L_{\mathrm{MST}}=(N-1) \sqrt{3} a .
$$

\section{CONDUIT LENGTH COMPARISONS BETWEEN STAR AND SMT ARCHITECTURES}

The ratio of the required conduit lengths for star and SMT architectures $L_{\mathrm{STAR}} / L_{\mathrm{SMT}}$ is shown in Fig. 7(a) for the case of hexagonal cells in a hexagonal layout with the CS in the center of the cell. The remarkable gain in using the SMT architecture instead of the star architecture is obvious. Moreover, the proportional cost for using the star architecture increases even more as the network grows; for instance, we observe from Fig. 7(a) that for $N=37, L_{\mathrm{STAR}} / L_{\mathrm{SMT}}$ is 2.51 , but for $N=271$, it is 6.69 .

In addition, the conduit length in a star architecture depends on the location of the CS in the cell; $L_{\mathrm{STAR}}$ yields a minimum value if the CS is in the center of the cell, but it may increase considerably if the CS is located in some other part of the cell. However, this is not the case for SMT and also for MST architectures, where the base can easily be placed in any convenient location without any increase in the conduit length. Therefore, $L_{\mathrm{STAR}} / L_{\mathrm{SMT}}$ further increases if the CS is not in the center of the cell. Let us consider, for instance, the extreme situation where the CS is in the corner. Fig. 8(a) and (b) shows the star and SMT architectures, respectively, for such a case; the corresponding MST architecture is also illustrated in Fig. 8(c). It is observed from Fig. 7(b) that for $N=91, L_{\mathrm{STAR}} / L_{\mathrm{SMT}}$ reaches a value of 6.60 if the CS is in the corner; this value is considerably higher than 3.89, which is the value for the case where the CS is in the center.

Furthermore, the conduit length in a star architecture also depends on the shape of the cell; for a fixed cell area, if the cell has the shape of a hexagon (which approximates a circle), then the required conduit length is less than that of any other cell shape. For the SMT architecture, however, the effect of cell shape on the conduit length is marginal, as described in Section III-C2. In Fig. 9, rectangular cells in the hexagonal 


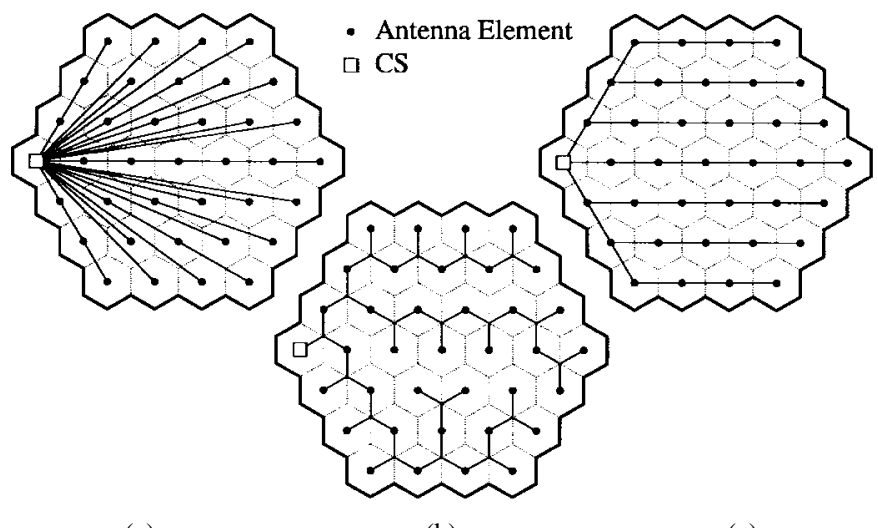

(a)

(b)

(c)

Fig. 8. (a) Star, (b) SMT, and (c) MST architectures for the case where the $\mathrm{CS}$ is in the corner of the cell.

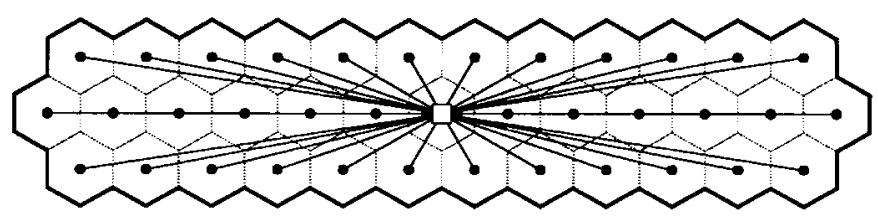

(a)

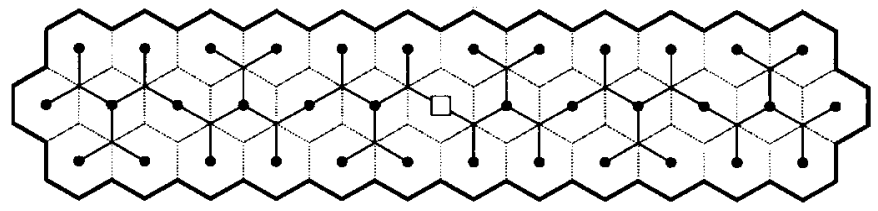

(b)

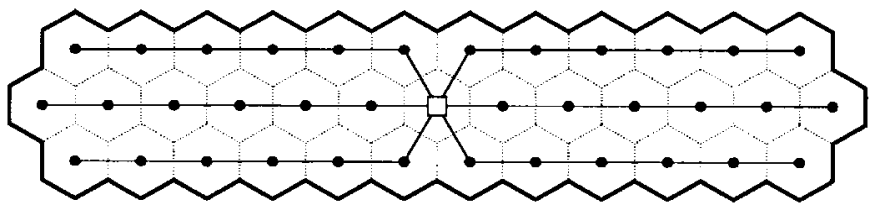

(c)

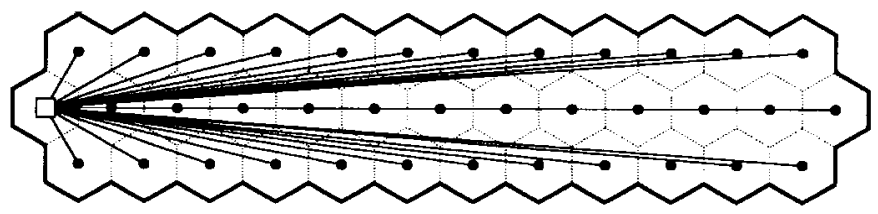

(d)

Fig. 9. Rectangular cells in hexagonal layout with $N=37$ antenna elements: (a) Star, (b) SMT, and (c) MST architectures with the CS in the center. (d) Star architecture with the CS in the corner.

layout with $N=37$ are illustrated. If the CS is in the center of the cell, then $L_{\mathrm{STAR}} / L_{\mathrm{SMT}}$ [see Fig. 9(a) and (b)] would be 3.80 [Fig. 7(c)]; this is considerably higher than the value 2.51 obtained from Fig. 7(a), which is the corresponding ratio for a hexagonal cell, again with $N=37$. In Fig. 9(c), an MST architecture, for which the conduit length is totally independent of the cell shape, is also shown.

Obviously, for star architecture, the combined effect of the cell shape and the CS location is even more severe on the conduit length. For instance, for the case illustrated in Fig. 9(d), where the CS is located in the corner of a rectangular cell with $N=37, L_{\mathrm{STAR}} / L_{\mathrm{SMT}}$ increases to 7.21 [Fig. 7(d)];

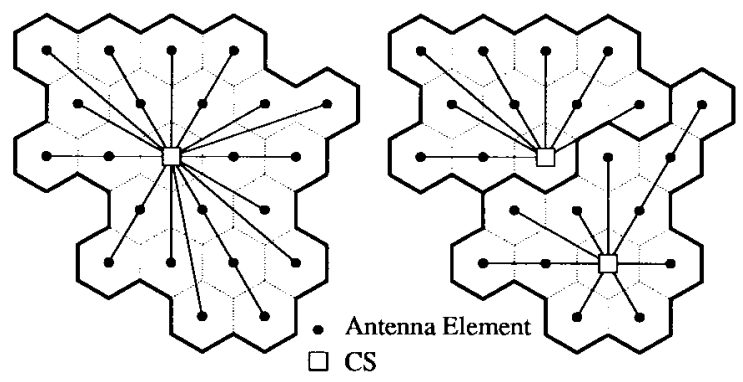

(a)
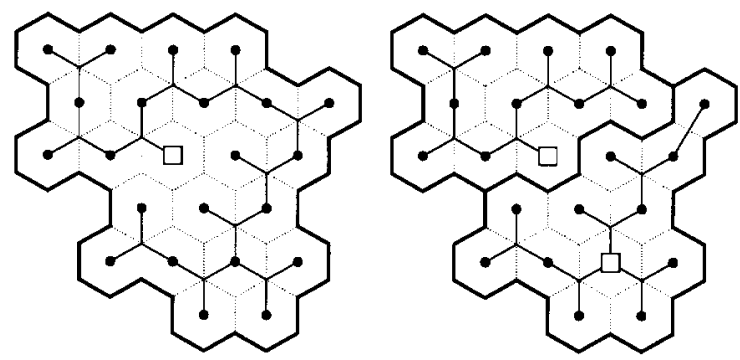

(b)

Fig. 10. Cell splitting for SMT architecture.

this value is significantly higher than 2.51 , which is the corresponding ratio for the case where the CS is in the center of a hexagonal cell.

If a cell grows too large, it may become beneficial or necessary to split it into two (or more) cells. In a star architecture, since all of the existing conduits meet in a central location, splitting a cell requires a major reconfiguration; therefore, it is not practical, as shown in Fig. 10(a) [28]. An SMT (also MST) architecture, however, can easily be split without any major reconfiguration [Fig. 10(b)].

\section{InterconNection of CENTRAL Stations}

In general, an access network infrastructure consists of antenna elements, CS's, main switches, and transmission links interconnecting them. In such a network, antennas are connected to the corresponding CS, and various CS's are connected to a main switch, which links the entire network to a fixed network such as the PSTN.

One way to establish this interconnection is to link a CS and the corresponding antenna elements in the form of an SMT, and then to connect the CS's and the main switch, again in an SMT form, as shown in Fig. 11(a). We call this structure a double-SMT architecture. Such a structure can be thought of as a tree with two levels, as illustrated in Fig. 11(b). It is worth noting that, although both levels of this interconnecting tree are constructed as SMT's, the overall tree is not an SMT, nor even an ST. Therefore, the length of a double-SMT architecture is (slightly) more than that of a single-SMT type. It is reported in the literature, however, that the double-star architecture is significantly shorter than the single-star type [10], [28]. Consequently, it is not a good strategy to construct a double-SMT architecture instead of a single-SMT type, unlike the case for the star architecture. Nevertheless, a double-SMT architecture is still shorter than a double-star type, simply because the SMT in each level of the tree is shorter than 


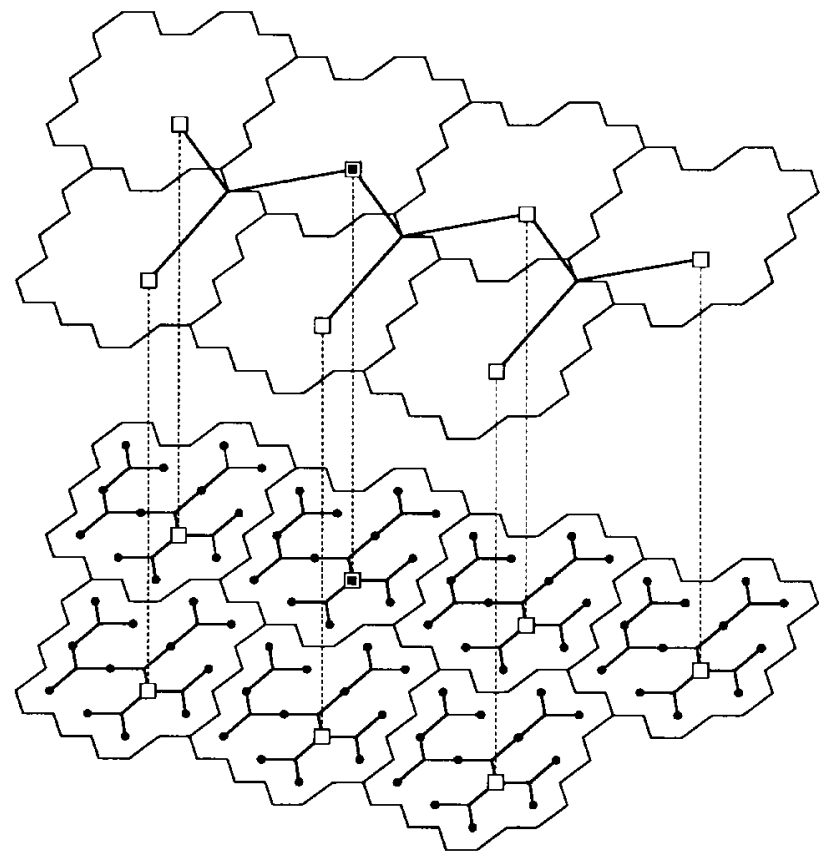

(a)

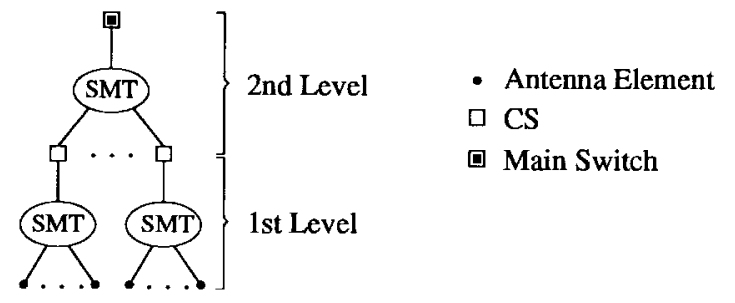

(b)

Fig. 11. Double-SMT architecture for interconnecting CS's in hexagonal layout. (a) Details of the architecture. (b) Two-level tree representation.

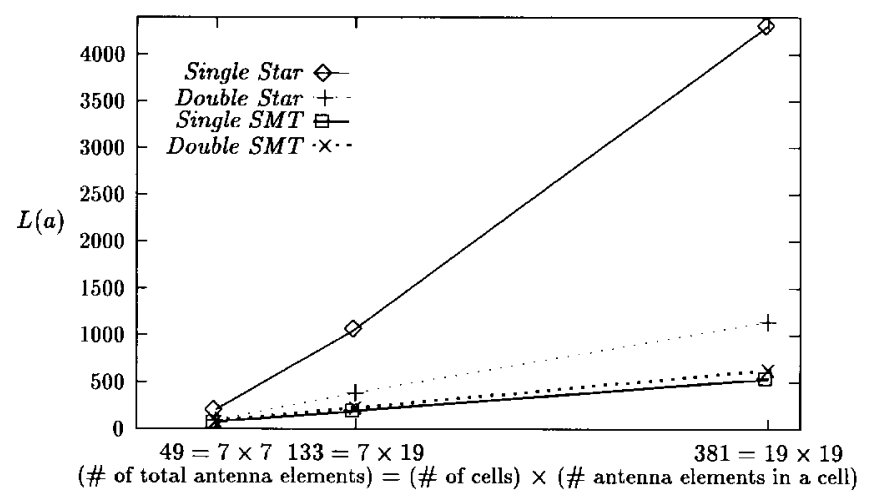

Fig. 12. Conduit length comparisons for single- and double-layer architectures.

the corresponding star type. Fig. 12 shows the conduit length comparisons, in terms of hexagon radius $a$, for the one- and two-level architectures, for the cases of seven cells with seven antenna elements, seven cells with 19 antenna elements, and 19 cells with 19 antenna elements. For all of these cases, both the cells and the structures formed by the combination of cells are in hexagonal shapes.

As far as the conduit length is concerned, an improved method of realizing the interconnection is shown in Fig. 13(a). (a)

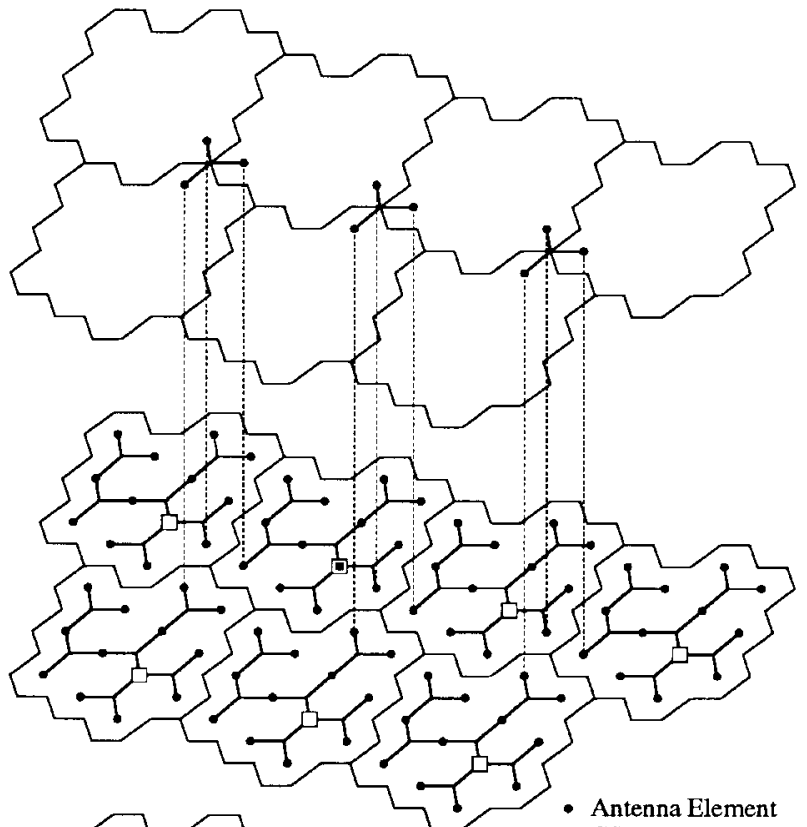

(b)

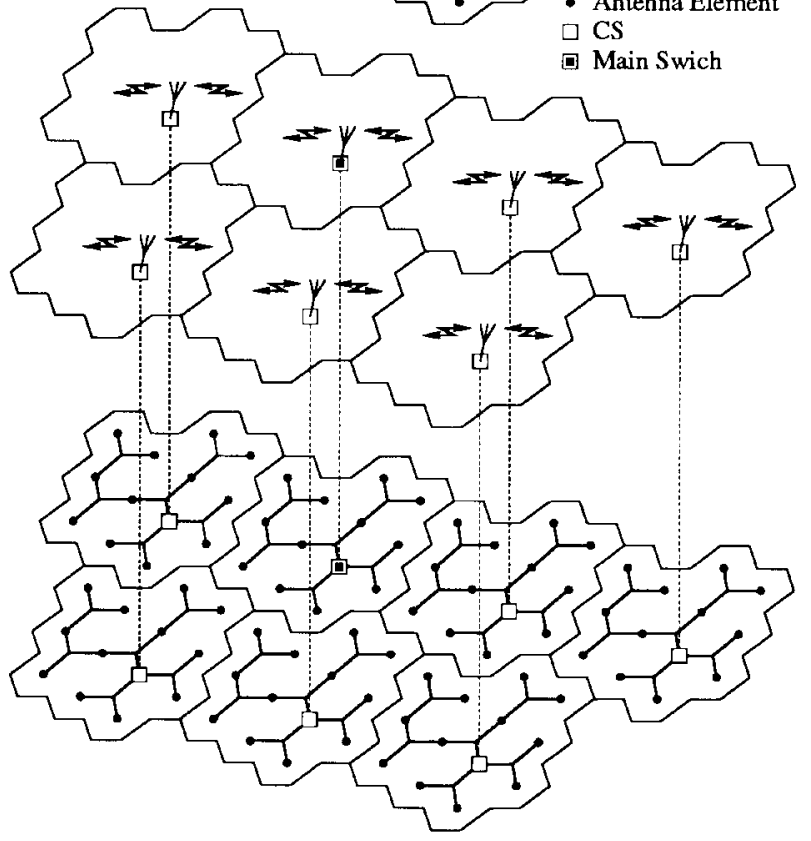

Fig. 13. (a) Extended SMT and (b) single-layer SMT architectures for interconnecting cells in hexagonal layout.

In this case, the overall structure is a single SMT, which we call an extended SMT architecture; hence, it yields the minimum total conduit length.

One other possibility is that the interconnection between the CS's and the main switch may be established via radio [Fig. 13(b)]. In such a case, the structure can be thought of as a collection of unconnected SMT's; therefore, the conduit length need only be considered for the connections in the first level of the tree shown in Fig. 11(a). Although this architecture, which we call a single-layer SMT, requires less conduit than the extended SMT type, there is the additional cost of the radio equipment to implement the second level of the tree illustrated in Fig. 11(a).

As an example, let us consider the case where a fixed area is served by $M$ cells, each having $N$ antenna elements. Using 


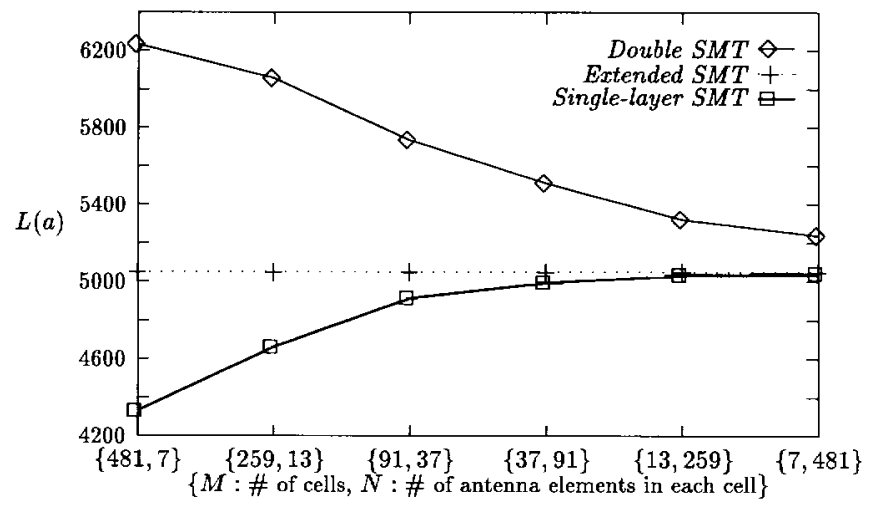

Fig. 14. Conduit length comparisons for various interconnection strategies with $M N=3367$.

(8), the required conduit lengths for the three architectures described above can be found, in terms of hexagon radius $a$, as

$$
\begin{aligned}
L_{\text {extended SMT }} & =\frac{3}{2}(M N-1) a \\
L_{\text {single-layer SMT }} & =\frac{3}{2} M(N-1) a \\
L_{\text {double SMT }} & =\frac{3}{2} M(N-1) a+\frac{3}{2}(M-1) \sqrt{N} a .
\end{aligned}
$$

The required conduit lengths are plotted in Fig. 14 for the case of $M N=3367$. It is observed from Fig. 14 that if a doubleSMT architecture has to be implemented, the best strategy would be to maximize the number of antenna elements in each cell (or, equivalently, to minimize the number of cells in the fixed service area); this would give a large $N$ and small $M$. However, if the CS's could be connected to the main switch via a radio link (single-layer SMT), then it would be most efficient to minimize the number of antenna elements in each cell (or, equivalently, to maximize the number of cells), hence yielding a small $N$ and large $M$.

\section{Vi. Cable Configurations in THE SMT CONDUIT ARCHITECTURE}

All of the logical topologies described in Section II can be realized within the optimal SMT conduit infrastructure. Fig. 15(a) shows the mapping of a wired network which has logical star topology into an SMT conduit architecture. This network may correspond to a fiber optic microcellular system utilizing SCM, or to an SDA system with one antenna element per sector. Physical implementation of a wired network with logical bus topology is illustrated in Fig. 15(b), which may represent a microcellular system with multilevel star links realized by using 1:2 passive splitters (located at the Steiner points) or a DA system. It is worth noting that both the conduit and cable lengths for the network shown in Fig. 15(b) are minimal since, in this case, the actual cable architecture is in SMT form as well. If we do not take the cost of the splitters (and possible amplifiers in the feeder) ${ }^{4}$ into the account, this architecture results in the minimal wired network infrastructure cost.

\footnotetext{
${ }^{4} \mathrm{~A}$ complete cost analysis should also take into consideration the costs of the other network elements, such as the antenna elements, couplers, splitters, and amplifiers.
}

(a)

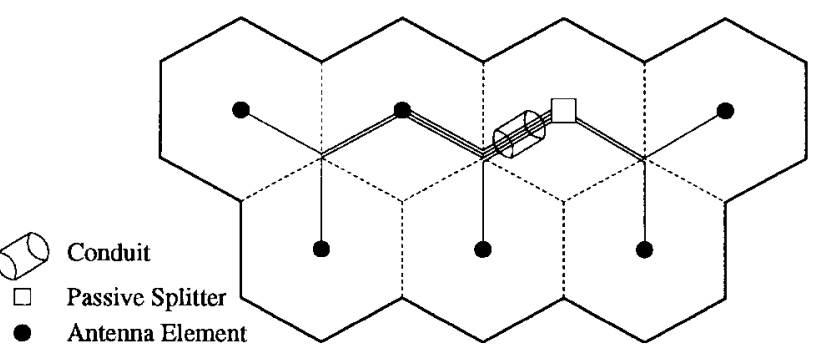

CS

(b)

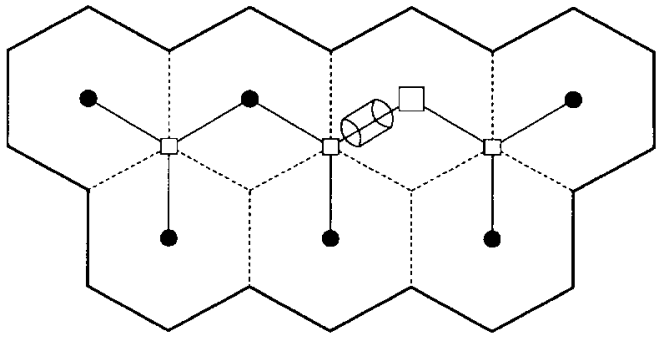

Fig. 15. SMT conduit architecture for an access network which has (a) logical star and (b) logical bus topology.

One other way of realizing the Steiner points is to use multifiber optical links. It is a common practice of manufacturers to package a large number of optical fibers within one cable because the manufacturing and installation costs for multifiber and single-fiber cables are not significantly different [29]. In such a case, there is no need for couplers and splitters, and consequently, no loss is induced at the Steiner points. Finally, we note that the conduit architectures presented in this paper are not limited to fiber links; either fiber optic or coaxial cables may be utilized in the conduit.

\section{CONCLUDING REMARKS}

In this study, some concepts from minimal network theory are utilized in order to determine cost-efficient as well as robust and flexible antenna interconnection architectures for PCS access networks.

The optimum SMT and the suboptimum MST architectures both result in a tremendous reduction in conduit length compared to the conventional star type, especially in large networks. The conduit length in a star architecture depends on both the service area shape and CS location in the service area. The conduit length for the SMT architecture, however, is independent of the CS location, and the effect of the service area shape on the conduit structure is marginal. For the MST architecture, these two factors do not have any effect at all on the conduit length. As a result, if the CS is not in the center of the service area and/or if the shape of the service area is not close to a circle, then the relative difference in conduit length between star and SMT (and also MST) architectures becomes even more significant. Furthermore, splitting a service area can be achieved with minimum network reconfiguration for the SMT and MST architectures, unlike the star type which requires major reconfiguration. Therefore, the SMT and MST architectures not only result in significantly less conduit, but they are also much more robust and flexible compared to the star type. Finally, the SMT (or MST) architecture can also be used for interconnecting various CS's in a network. 
The SMT's that are considered in this study are formed for hexagonal layout. In practice, though, the layout almost never has a regular shape. However, even if this were the case, to install the conduit according to an SMT structure may not be practical. Nevertheless, it is quite important to have a general understanding of the interconnection architectures. For an arbitrary layout, the most reasonable strategy may be to use an MST structure; because, as mentioned earlier, there are straightforward algorithms for constructing an MST, however, it may not be possible to construct the SMT as it is an NPcomplete problem. Although there is an increase in the conduit length of the MST architecture over that of the SMT type of at most $15 \%$, both architectures still offer substantial savings in conduit length, along with flexibility and robustness, compared to the star architecture.

\section{REFERENCES}

[1] M. Shibutani, T. Kanai, W. Domon, K. Emura, and J. Namiki, "Optical fiber feeder for microcellular mobile communication system (H-015)," IEEE J. Select. Areas Commun., vol. 11, pp. 1118-1126, Sept. 1993.

[2] D. Tang, "Fiber-optic antenna remoting for multisector cellular cell sites," in Proc. IEEE Int. Conf. Commun., vol. 1, 1992, pp. 76-81.

[3] L. J. Greenstein, N. Amitay, T.-S. Chu, L. J. Cimini, Jr., G. J. Foschini, M. J. Gans, C.-L. I, A. J. Rustako, Jr., R. A. Valenzuela, and G. Vanucci, "Microcells in personal communications systems," IEEE Commun. Mag., pp. 76-88, Dec. 1992.

[4] J. Sarnecki, A. Javed, P. O'Kelly, and K. Dick, "Microcell design principles," IEEE Commun. Mag., pp. 76-82, Apr. 1993.

[5] K. Morita and H. Ohtsuka, "The new generation of wireless communications based on fiber-radio technologies," IEICE Trans. Commun., vol. E76-B, pp. 1061-1068, Sept. 1993.

[6] H. Jung and O. K. Tonguz, "Access network for PCS," in Proc. IEEE 7th Int. Symp. Personal, Indoor, and Mobile Radio Commun., vol. 3, 1996, pp. 1267-1271.

[7] J. C. Fan, C. L. Lu, R. F. Kalman, and L. G. Kazovsky, "Design and analysis of a novel fiber-based PCS optical network," in Proc. IEEE Int. Conf. Commun., vol. 1, 1995, pp. 601-605.

[8] O. K. Tonguz and H. Junk, "Personal communications access networks using subcarrier multiplexed optical links," J. Lightwave Technol., vol. 14, pp. 1400-1409, June 1996.

[9] W. I. Way, "Optical fiber-based microcellular systems: An overview," IEICE Trans. Commun., vol. E76-B, pp. 1091-1102, Sept. 1993.

[10] K. Kumozaki, "A fiber-optic passive double star network for microcellular radio communication systems applications," IEICE Trans. Commun., vol. E76-B, pp. 1122-1127, Sept. 1993.

[11] H. Harada, S. Kajiya, K. Tsukamoto, S. Komaki, and N. Morinaga, "TDM intercell connection fiber-optic bus link for personal radio communication systems," IEICE Trans. Commun., vol. E78-B, pp. 1287-1294, Sept. 1995.

[12] A. Salmasi and K. Gilhousen, "On the system design aspect of code division multiple access (CDMA) applied to digital cellular and personal communications networks," in Proc. 41st IEEE Veh. Technol. Conf., 1991, pp. 57-62.

[13] T. S. Chu and M. J. Gans, "Fiber optic microcellular radio," in Proc. 41st IEEE Veh. Technol. Conf., 1991, pp. 339-344.

[14] A. J. Viterbi and R. Padovani, "Implications of mobile cellular CDMA," IEEE Commun. Mag., pp. 38-41, Dec. 1992.

[15] H. Yanikomeroglu and E. S. Sousa, "CDMA distributed antenna system for indoor wireless communications," in Proc. IEEE 2nd Int. Conf. Universal Personal Commun., vol. 2, 1993.

[16] E. S. Sousa, "Antenna architectures for CDMA integrated wireless access networks," in Proc. IEEE 6th Int. Symp. Personal, Indoor, and Mobile Radio Commun., vol. 3, 1995, pp. 921-925.

[17] D. W. Tcha and M. G. Yoon, "Conduit and cable installation for a centralized network with logical star-star topology," IEEE Trans. Commun., vol. 43, pp. 958-967, Feb./Mar./Apr. 1995.

[18] K. H. Rosen, Discrete Mathematics and Its Applications, 3rd ed. New York: McGraw-Hill, 1995.
[19] A. O. Ivanov and A. A. Tuzhilin, Minimal Networks: The Steiner Problem and Its Generalization. Boca Raton, FL: CRC Press, 1994

[20] F. K. Hwang and D. S. Richards, "Steiner tree problems," Networks, vol. 22, pp. 55-89, 1992.

[21] F. K. Hwang and J. F. Weng, "Hexagonal coordinate systems and Steiner minimal trees," Discrete Math., vol. 62, pp. 49-57, 1986.

[22] Z. A. Melzak, Companion to Concrete Mathematics. New York: John Wiley, 1973 .

[23] _ "On the problem of Steiner," Can. Math. Bull., vol. 4, pp. 143-148, May 1961.

[24] H. O. Pollak, "Some remarks on the Steiner problem," J. Combinatorial Theory, ser. A 24, pp. 278-295, 1978.

[25] F. Chung and R. Graham, "Steiner trees for ladders," Ann. Discrete Math., vol. 2, pp. 173-200, 1978.

[26] M. R. Garey, L. R. Graham, and D. S. Johnson, "The complexity of computing Steiner minimal trees," SIAM J. Appl. Math., vol. 32, pp. 835-859, 1977.

[27] F. K. Hwang and D. Z. Du, "Steiner minimal trees on Chinese checkerboards," Math. Mag., vol. 64, pp. 332-339, Dec. 1991.

[28] K. A. Falcone and O. K. Tonguz, "Practical constraints in growth of lightwave networks," IEEE Trans. Commun., vol. 44, pp. 348-355, Mar. 1996.

[29] J. Bannister, M. Gerla, and M. Kovacevic, "An all-optical multifiber tree network,” J. Lightwave Technol., vol. 11, pp. 997-1008, May/June 1993.

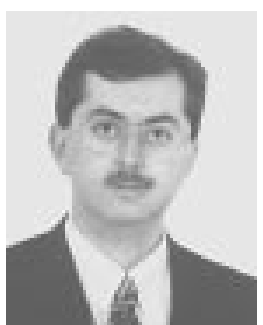

Halim Yanikomeroglu was born in Giresun, Turkey, in 1968. He received the B.Sc. degree in electrical and electronics engineering from the Middle East Technical University, Ankara, Turkey, and the M.A.Sc. degree in electrical engineering from the University of Toronto, Toronto, Ont., Canada, in 1990 and 1992, respectively. Since September 1994, he has been working toward the Ph.D. degree at the Department of Electrical and Computer Engineering, University of Toronto.

From January 1993 to July 1994, he was employed by the Research and Development Group of Marconi Kominikasyon A.S., Ankara, Turkey. From September 1990 to August 1992 and from September 1994 to the present, he has been employed as a Research Assistant, and also as a Teaching Assistant from January 1991 to May 1992 and from January 1995 to the present, at the University of Toronto. In the summer of 1996, he taught a course on telecommunication transmission theory to engineers of Ontario Hydro, Toronto. His current research interests include wireless access to fixed networks, distributed antenna systems, and diversity techniques.



Elvino S. Sousa (S'79-M'86-SM'96) received the B.A.Sc. degree in engineering science and the M.A.Sc. degree in electrical engineering from the University of Toronto, Toronto, Ont., Canada, in 1980 and 1982, respectively, and the Ph.D. degree in electrical engineering from the University of Southern California, Los Angeles, in 1985.

Since 1986, he has been with the Department of Electrical and Computer Engineering, University of Toronto, where he is presently an Associate Professor. From 1986 to 1996, he was a Natural Sciences and Engineering Research Council of Canada (NSERC) University Research Fellow. He has performed research in the areas of packet radio networks and spread spectrum systems since 1983. His current interests are in the areas of spread spectrum systems, mobile communications, indoor wireless communications, and wireless LAN's. At the University of Toronto, he teaches courses in mobile communications and error control codes. Currently, he has research projects on the performance of microcellular networks, wireless multimedia networks, and wireless LAN's. He has given many short courses and lectures on the topics spread spectrum and wireless communications in a number of different countries.

Dr. Sousa is an Associate Editor in the area of CDMA systems for the IEEE Transactions on Communications and was the Technical Program Chairman for PIMRC'95. 